auf eine Strahlenbehandlung untersuchten Lebensmittel lag in den Jahren 1993 bis 1995 unter $2 \%$; 1996 ist der Prozentsatz auf 3,1\% (lediglich als Trend zu sehen) angestiegen.

Auffällig ist der im Verhältnis zu den früheren Jahren hohe Anteil positiver Befunde bei Gewürzen, Gewürzmischungen und Gewürzzubereitungen. Speziell Gewürzzubereitungen wiesen bestrahlte Bestandteile auf. Auch bei den Krusten-, Schalen- und Weichtieren konnten kontinuierlich bestrahlte Proben festgestellt werden.
Der Nachweis bei Lebensmitteln, in denen bestrahlte Zutaten enthalten sind oder in denen dieselbe Zutat sowohl als bestrahlt als auch unbestrahlt eingesetze wird, ist wesentlich schwieriger als der Nachweis direkt bestrahlter Lebensmittel. Durch die Weiterentwicklung der analytischen Methoden wurden immer häufiger derartige Lebensmittel untersucht, wie z. B. Frischkäse mit Pfefferbzw. Schnittlauchumhüllungen, Backwaren etc.

Es stellt sich die Frage, ob tatsächlich immer mehr bestrahlte Gewürze, Ge- würzmischungen und Gewürzzubcreitungen in den Verkehr gebracht werden oder ob eine gezielte Probenahme und differenziertere Analytik für die höhere Nachweisquote der positiven Befunde verantwortlich sind.

Danksagung

Ich danke den Chemischen- und Lebensmitteluntersuchungsämtern, die mir die benötigten Uncersuchungsergebnisse für die durchgeführte Auswerrung zur Verfügung gestellt haben.

Anschrift der Verfasserin:

Karina Mainczyk, Chemisches und Lebensmitteluntersuchungsamt der Landeshauptstadt Düsseldorf, Lamberrusstr.1, 40213 Düsseldorf

\title{
Influenza-Aktivität im Winter 1996/97
}

\section{Einleitung}

Eine mittelschwere Epidemie zum Jahreswechsel 1995/96 hat die Influenza wieder mehr ins Bewußtsein einer breiteren Öffentlichkeit gerückt. Die durchschnittliche Zahl von Todesfällen wegen Erkrankungen der Atemwege wurde allein im Januar 1996 um erwa 3300 Todesfälle überschritten. Zudem verdeutlicht auch der Anstieg bei Hospitalisierungen wegen respiratorischer Infekte bei über 60jährigen von etwa 0,8 auf bis zu 2,5 Prozent während der epidemischen Influenzaaktivität die Gefährlichkeit der Influenza. Insgesamt sind im Januar und Februar 1996 bundesweit etwa bis zu 30000 Todesfälle über das normale Maß hinaus (Todesursachenstatistik) registriert worden. Die Komplikationen und Sekundärinfektionen waren nicht nur auf den Respirationstrakt beschränkt, sondern es wurden auch vermehrt Meningitiden, Myokarditiden, Stoffwechselentgleisungen u. v. m. beobachtet.

Das Beobachtungssystem der Arbeitsgemeinschaft Influenza (AGI) mit bundesweit etwa 600 allgemeinmedizinischen, internistischen und pädiatrischen Sentinelpraxen beobachtet die Influenza-Aktivität in den Winterhalbjahren seit 1992/93. Den wesentlichen Bestandteil des Systems stellen die unentgeltlich mitarbeitenden Meldepraxen dar. Hier werden die akuten respiratorischen $\mathrm{Er}$ krankungen (ARE) in fünf Altersgruppen erfaßt. Informationen zur Schwere der Erkrankung werden durch die Zahl der Arbeitsunfähigkeitsmeldungen (AU) - bei Kindern und Rentnern Erkrankungen, die häusliche Pflege erfordern, - der Hospitalisierungen und der Todesfälle aufgrund akuter respiratorischer Erkrankungen in den jeweiligen Altersgruppen ermittelt. Als Bezugsgröße dient die Anzahl der Praxiskontakte. Neben diesen Beobachtungen zur Morbidität sind jedoch auch Isolierungen und Feintypisierungen sowie sonstige Erregernachweise unerläßlich. Proben dazu stammen überwiegend aus Praxen, die auch Morbiditätsdaten ermitteln.

Aufgrund der guten Zusammenarbeit und Kooperationsbereitschaft der nationalen Referenzzentren für Influenza, vieler Landeshygieneinstitute und -untersuchungsämter sowie einiger Kliniken und privater Laboratorien konnten die Antigen-Diagnostik und Virusisolierung intensiviert werden.

Daneben spielt das Laborberichtssystem für respiratorische Erreger am Robert Koch-Institut eine wichtige Rolle als Referenzsystem. Hier werden Antikörpernachweise aus einzelnen und gepaarten Seren in einigen Hygieneinstituten und Laboren in den neuen Ländern zusammengefaßt [1].

Darüber hinaus unterhält die AGI einen Datenaustausch mit einer Vielzahl von europäischen und nichteuropäischen Ländern sowie mit der Weltgesund- heitsorganisation (WHO). Als Mitglied des European-Influenza-SurveillanceScheme (EISS), einem Zusammenschluß von nunmehr neun nationalen Beobachtungssystemen, ist die AGI auch eng in die internationale Surveillance eingebunden [2].

\section{Beobachtungsergebnisse für den Winter 1996/97}

\section{Nachbarländer}

In der Beobachtungsphase 1996/97 zeigten erste Isolierungen um die 40. Kalenderwoche (KW) 1996 in Portugal, Spanien und Frankreich eine sporadische Influenza-Aktivität an, die überwiegend durch Viren des Typs $A(H 3 N 2)$ verursacht wurde. Spürbare Morbiditätserhöhungen und erste nennenswerte Influenza-Ausbrüche wurden in Westeuropa in Portugal, Spanien und Frankreich etwa um die $47 . \mathrm{KW}$ registriert. Es folgten England in der 49. KW, Belgien ab etwa der 50. KW und die Niederlande in der 51. KW.

Die Influenza-Aktivität begann im Süden in der Schweiz im Grenzbereich zu Südfrankreich. Es wurden ab der 44 . KW erste Influenzaviren isoliert. Ein Morbiditätsanstieg zeigte sich in der 49 . KW, und in der 51. KW wurde die epidemische Schwelle überschritten. In Österreich stieg die Morbidität an respiratorischen Erkrankungen in der 51. und 52. KW leicht an. Deutliche Morbiditätssteigerungen machten sich erst etwa in der zweiten bis dritten KW 1997 be- 
merkbar. Der Peak in Österreich lag etwa in der fünften bis sechsten KW. Die Isolierungen aus Italien wiesen auf einen ähnlichen Verlauf hin.

In Rußland, speziell in den östlichen Landesteilen, wurde ebenfalls bereits in der 47. KW eine Influenza-A(H3N2)Aktivität beobachtet. In Rußland traten in einigen Städten recht hohe Morbiditäten auf. Die Epidemie erreichte etwa in der zweiten $K W$ auch die westlichen Teile Rußlands. In Polen wurde ähnlich wie in der Tschechei etwa ab der dritten $\mathrm{KW}$ eine erhöhte Influenza-Aktivität deutlich.

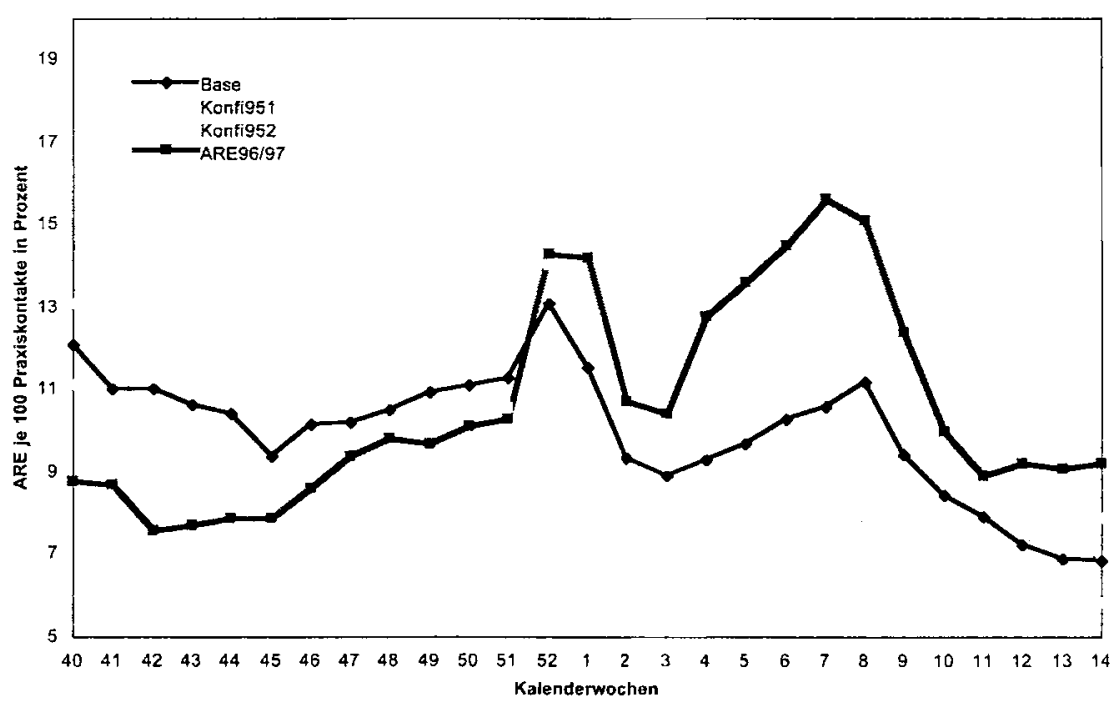

Abbildung 1: Basislinie und ARE-Anteile an den Praxiskontakten 1996/97.

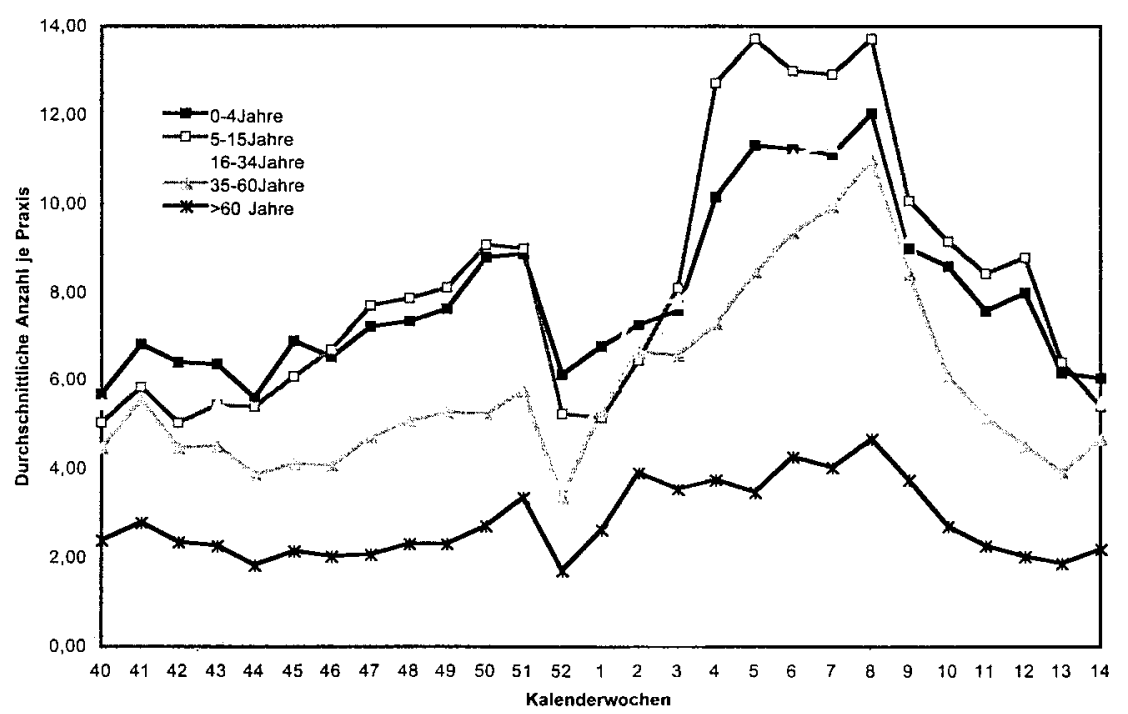

Abbildung 2: Durchschnitt der ARE pro Praxis in den Altersgruppen während der Beobachtungsperiode 1996/97.
Auch im Norden Europas (Dänemark, Norwegen, Finnland und Schweden) lag der Beginn der meßbar erhöhten Influenza-Aktivität etwa in der zweiten bis vierten KW. Der Peak wurde hier etwa in der fünften $\mathrm{KW}$ erreicht.

\section{Deutschland} gend auf die Kurven in den Abbildungen 1 und 2 sowie auf Telefonate mit Praxen, die auffällige Steigerungen der registrierten Erkrankungszahlen auf wiesen. Da in Abbildung 1 die Zahl der registrierten ARE auf die Praxiskontakte bezogen wird, kommt es bei redu-
Der folgende Text stützt sich überwie-

zierten Praxiskontaktzahlen, was insbesondere in der Weihnachts- und Neujahrswoche der Fall ist, zu einer Erhöhung der Werte. Bei den durchschnittlichen Zahlen pro Praxis beobachtet man in diesen Phasen einen Rückgang der Erkrankungszahlen. Erst beide Kurven zusammen erlauben in diesem Zeitraum eine Interpretation der Daten.

$\mathrm{Zu}$ Beginn der Beobachtungsphase 1996/97 war die Morbidität auffallend gering und lag deutlich unter der Basislinie.

Erste Isolierungen von Influenza A(H3N2) in der 49. KW 1996 bestätigten eine sporadische Aktivität von Influenza, auf die von der AGI bereits in der 48. KW hingewiesen worden war. In der 50. und 51. KW gab es in einigen westlichen Regionen der Bundesrepublik auch eine spürbare Morbiditätserhöhung besonders bei Kindern und Jugendlichen, die aufgrund fehlender Virusisolierungen jedoch nicht eindeutig mit Influenza in Verbindung gebracht werden konnte. Die AGI hatte jedoch bereits in der 50 . KW wegen der Gesamtsituation auf die hohe Wahrscheinlichkeit einer steigenden Influenza-Aktivität in den darauffolgenden Wochen aufmerksam gemacht.

In der 52. KW wurde der Basiswert erstmals überschritten. Die besonders bei Kindern und Jugendlichen in dieser Woche jedoch rückläufigen Erkrankungszahlen (Weihnachtsferien) deuteten auf einen verzögerten Beginn der Influenzawelle hin (Abb. 2). Dies wurde in der 51. $\mathrm{KW}$ bereits von der AGI mitgeteilt. Ein Anstieg der Erkrankungszahlen war in der ersten und zweiten $\mathrm{KW}$ besonders im Westen und Südwesten der Bundesrepublik zu beobachten. Die klinischen Bilder wiesen aber eher auf eine Mischätiologie hin, und es wurden nur vereinzelt Häufungen von influenzatypischen Erkrankungen beobachtet (AGI-Wochenbericht 1/97). Bereits in der zweiten $\mathrm{KW}$ wurde auf eine erneut steigende Influenza-Aktivität hingewiesen. Ab der dritten $\mathrm{KW}$ beobachtete die AGI auch in den östlichen Landesteilen, besonders in Brandenburg und Berlin, eine lokale Influenza-Aktivität. Erst in der vierten $K W$ signalisierte ein sprunghafter Anstieg der ARE-Morbidität bei Kindern und Jugendlichen den eigentlichen Beginn der Influenzawelle.

Ab der fünften KW gab es erste Anzeichen für ein Sistieren des Anstiegs bei Kindern und Jugendlichen, eine generell 
nur langsame Morbiditätserhöhung bei den Erwachsenen und das weitgehende Fehlen eines Anstieges bei älteren Personen (AGI-Wochenbericht 5/97). Nach dem Peak etwa in der siebten bis achten $\mathrm{KW}$ ging die Morbidität innerhalb weniger Wochen auf ein fast normales Niveau zurück. Gegen Ende der Beobachtungsphase blieb das Verhältnis der ARE zu den Praxiskontakten leicht erhöht und stieg in einigen Regionen besonders bei Kindern und Jugendlichen nochmals an. Es gab keine Anzeichen für erneute großflächige Influenza-Ausbrüche, und die Beobachtungsphase wurde zu Ostern (15. KW) beendet. Dennoch traten in der Folge bis in den April und Mai Häufungen von influenzaähnlichen Erkrankungen auf (besonders in Mecklenburg-Vorpommern, Bayern, Baden-Württemberg und Sachsen), die teils auch durch Isolierungen als influenzabedingt bestätigt werden konnten. Insgesamt blieb die InfluenzaAktivität deutlich hinter der im Winter 1995/96 beobachteten zurück.

Für den Zeitraum der maximalen Influenza-A- und -B-Aktivität (vierten bis neunten KW 1997) können (bei ausreichender Repräsentativität der AGI-Praxen) aus den durchschnittlichen Erkrankungen je Praxis jedoch etwa fünf Millionen akute respiratorische Erkrankungen, die zu Arztbesuchen führten, über das normale Maß hinaus angenommen werden.

\section{Altersgruppen}

Bei Kleinkindern (bis vier Jahre) wurde eine ähnlich hohe Morbidität wie im Winter 1995/96 erreicht. Im Vergleich zu den vergangenen Jahren war die Morbidität in dieser Altersgruppe gegenüber den Fünf- bis 15jährigen in diesem Jahr recht hoch, und es ließen sich zwei Gipfel erkennen, wobei der eine in die Phase der maximalen Influenza-A(H3N2)und der höhere in die Phase der höchsten Influenza-B-Aktivität fiel. Bei den Fünf-bis 15jährigen wirkte sich die Influenza-Aktivität wie in den vorangegangenen Jahren sehr deutlich aus. Auch hier lagen die Morbiditäten während $\operatorname{der} \mathrm{A}(\mathrm{H} 3 \mathrm{~N} 2)$ - und -B-Aktivität ähnlich hoch. In dieser Altersgruppe war der Rückgang der Morbidität über die Weihnachts- und Neujahrsfeiertage am deutlichsten (Abb. 2).

Die höheren Altersgruppen, insbesondere die über 60jährigen, wurden deutlich geringer erfaßt, was besonders im Vergleich zu der Epidemie im Winter 1995/96 auffällig ist. Die Morbidität in

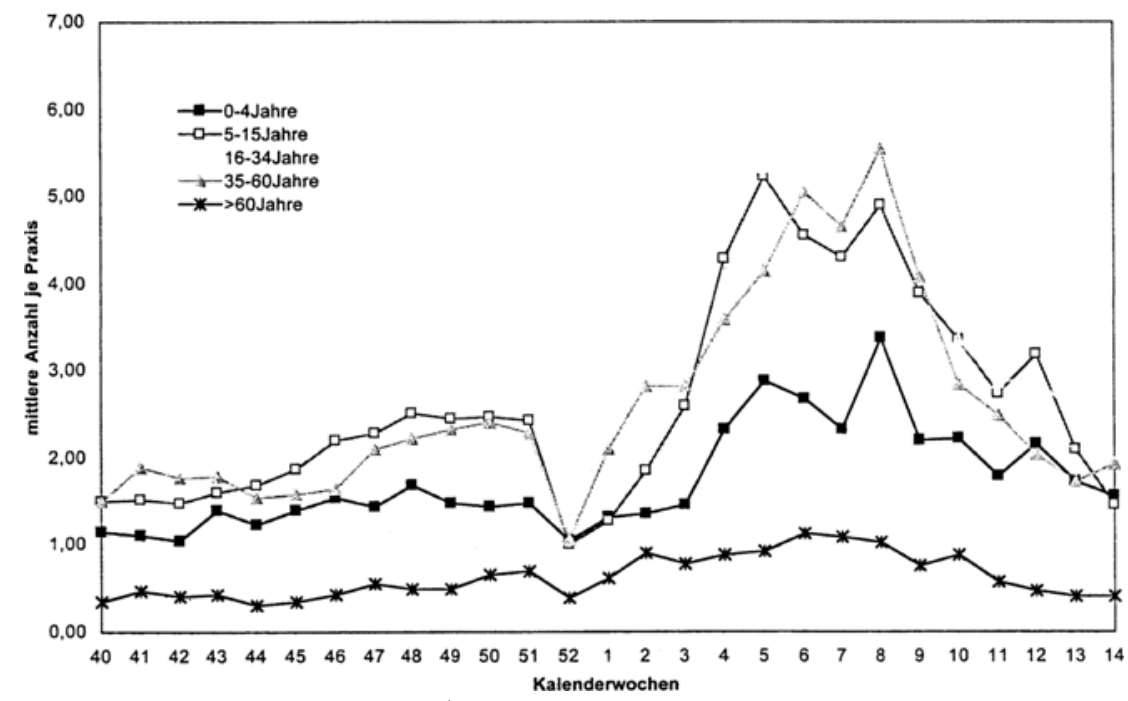

Abbildung 3: Durchschnitt der im Winter 1996/97 registrierten »Arbeitsunfähigkeiten * pro Praxis nach Altersgruppen.

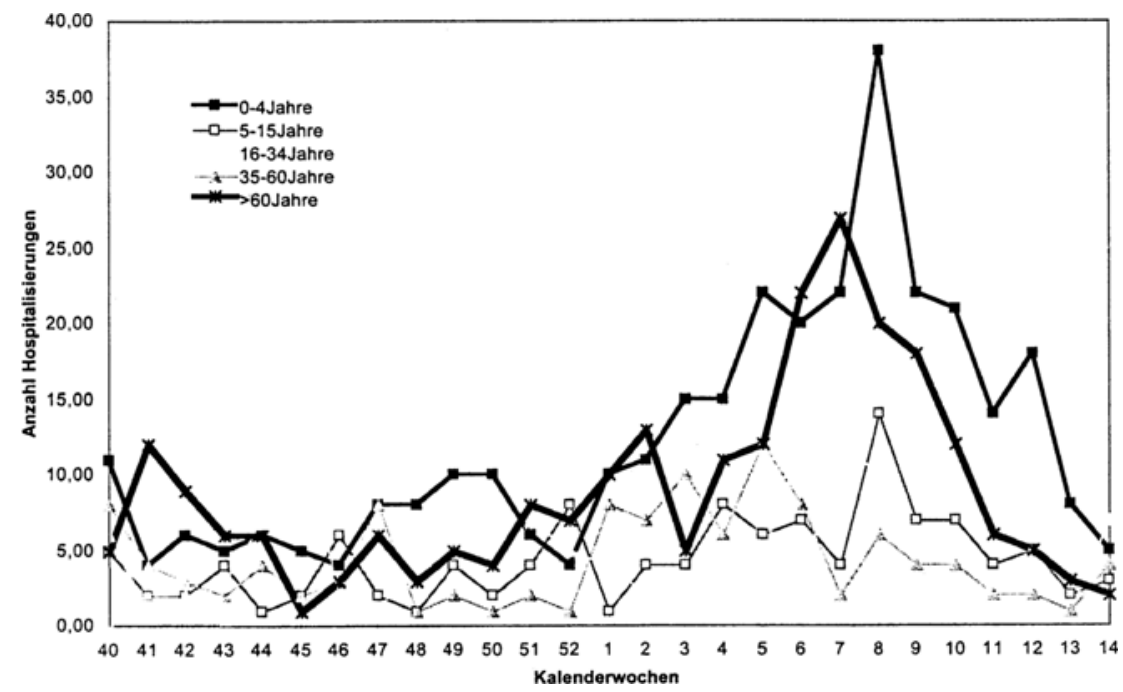

Abbildung 4: Im Winter 1996/97 bei AGI-Praxen registrierte Hospitalisierungen aufgrund respiratorischer Infekte in den Altersgruppen.

diesen Altersgruppen war in der Phase der überwiegenden Influenza-B-Aktivität am höchsten.

\section{Arbeitsunfähigkeiten}

Insgesamt verliefen die registrierten $A U$ aufgrund respiratorischer Infekte nahezu parailel zur Menge der Erkrankungen. Während der Phase erhöhter Influenza-Aktivität stiegen die registrierten AU deutlich an. Im Zeitraum von der vierten bis neunten $K W$ können in allen Altersgruppen etwa 2,3 Millionen AU über das normale Maß hinaus angenommen werden (ausgehend von den durchschnittlich registrierten AU je Praxis). Diese wurden überwiegend in den Al- tersgruppen der 16- bis 60jährigen, also der arbeitenden Bevölkerung, beobachtet (Abb. 3).

In den Altersgruppen der 16- bis 60jährigen liegt der Anteil der ärztlich erfaßten Arbeitsunfähigkeiten an den registrierten ARE bei etwa 40 Prozent und stieg während der Phase erhöhter Influenza-Aktivität auf etwa 50 Prozent an. Der Anteil der Arbeitsunfähigkeiten bei den 16- bis 35jährigen war dabei etwas höher und der Anstieg während der Influenzawelle etwas geringer. In der Altersgruppe der Fünf-bis 15jährigen betrug der Anteil der "Arbeitsunfähigkeiten « etwa 30 Prozent und stieg bis auf 


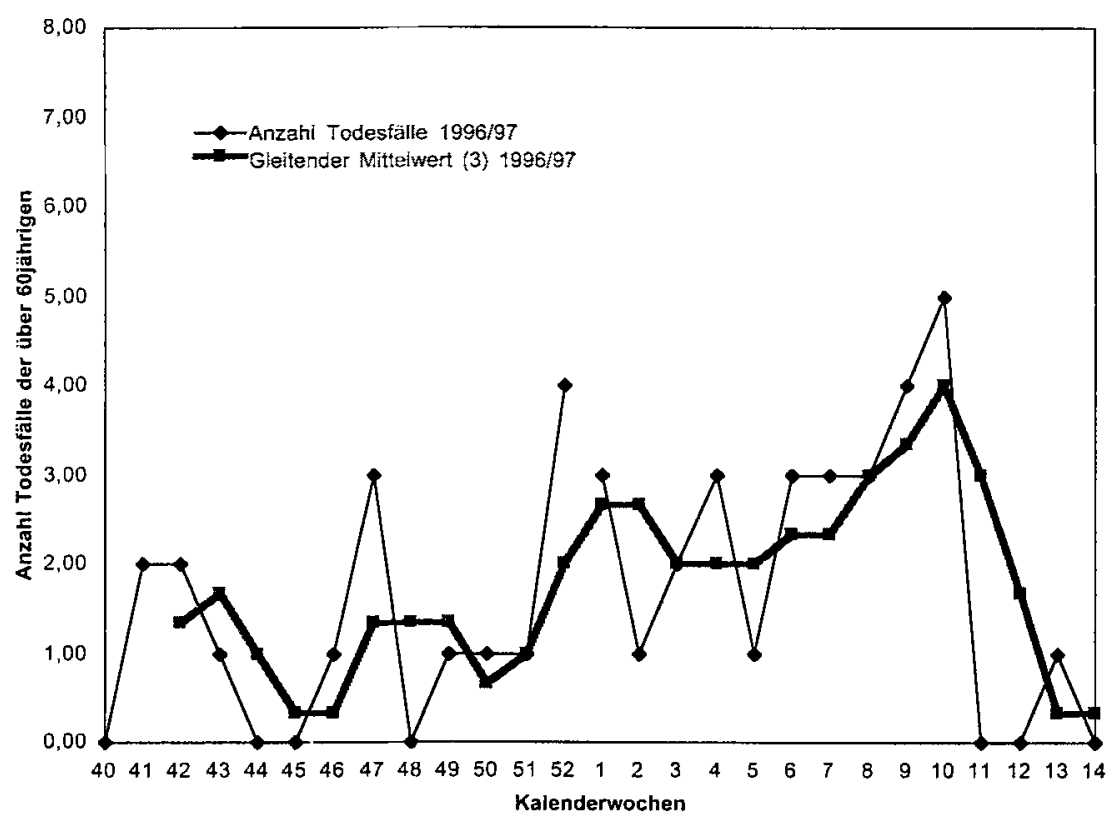

Abbildung 5: Im Winter 1996/97 bei AGI-Praxen registrierte Todesfälle aufgrund respiratorischer Infekte der über 60jährigen.

fast 40 Prozent. Bei den bis Vier- und über 60jährigen wurden etwa 20 Prozent Fälle mit kurzzeitiger häuslicher Pllege registriert und ein Anstieg auf etwa 25 Prozent während der vierten bis neunten KW 1997.

\section{Hospitalisierungen}

Die Zahl der registrierten Hospitalisierungen stieg während der Phase erhöhter Influenza-Aktivität deutlich an. Aufgrund der Steigerung der registrierten Hospitalisierungen pro Praxis über das normalerweise in diesem Zeitraum beobachtete Niveau hinaus, können in der vierten bis neunten $\mathrm{KW}$ etwa 30000 zusätzliche Hospitalisierungen aufgrund respiratorischer Erkrankungen in allen Altersgruppen geschätzt werden (Abb. 4).

Bei der Zahl der registrierten Hospitalisierungen war bei den Fünf- bis 60jährigen nur ein geringer Einfluß der Influenza erkennbar. Da eine Hospitalisierung in diesen Altersgruppen ein seltenes Ereignis darstellt, ist die Streuung groß. Der Anteil der Hospitalisierungen an den ARE, also gewissermaßen das Risiko schwerer zu erkranken, blieb in diesen Altersgruppen im gleitenden Mittelwert über fünf Wochen über den Beobachtungszeitraum etwa gleich und lag deutlich unter 0,3 Prozent.

Typisch dagegen ist der Anstieg der registrierten Hospitalisierungen bei den tersgruppe ähnlich viele Hospitalisierungen aufgrund von ARE registriert wie bei den über 60jährigen, und dies über einen längeren Zeitraum. Da die Erkrankungszahlen bei den bis Vierjährigen wesentlich höher waren, stieg die Hospitalisierungsrate nur auf etwas über 0,5 Prozent im Mittel der vierten bis neunten $\mathrm{KW}$ an. Die Spitzenwerte lagen in dieser Altersgruppe bei 0,7 Prozent.

\section{Todesfälle}

In diesem Jahr war ein moderater Anstieg der registrierten Todesfälle erkennbar, ähnlich wie im März 1995, als die Influenzawelle ebenfalls die über 60jährigen nur in geringerem $\mathrm{Maße}$ erfaßt hatte. Da Todesfälle aufgrund akuter respiratorischer Erkrankungen noch weit seltener registriert werden als Hospitalisierungen, ist die Streuung entsprechend höher. Der gleitende Mittelwert (drei Wochen) verdeutlicht aber dennoch den leichten Anstieg zum Jahreswechsel und das etwas erhöhte Niveau während der Influenza-Aktivität. In den anderen Altersgruppen war kein signifikanter Anstieg der Todesfälle erkennbar (Abb. 5). uber 60jahrigen. Er war einerseits d einen Anstieg der Erkrankungen bedingt, andererseits aber vor allem durch das besonders in dieser Altersgruppe hohe Risiko einer schwereren Erkrankung oder Komplikation, die eine Hospitalisierung erforderlich macht. Während der Influenzawelle stieg die Hospitalisierungsrate auf Spitzenwerte von fast zwei Prozent an. Die Steigerung der registrierten Hospitalisierungen bei den bis Fünfjährigen ist in diesem Jahr im Vergleich zu der Epidemie von 1995/96 auffällig hoch. Es wurden in dieser Al-

\section{Isolierungen}

Erste Isolierungen von Influenza $A(H 3 N 2)$ in der $49 . \mathrm{KW}$ und von $\mathrm{A}(\mathrm{H} 1 \mathrm{~N} 1)$ in der $50 \mathrm{KW}$ bestätigten die sporadische Aktivität in diesem Zeitraum. $\mathrm{Da}$ die $Z$ ahl der eingesandten und untersuchten Proben über Weihnachten und Neujahr zurückging, konnte ein erster Anstieg der Influenza-Aktivität in diesem Zeitraum besonders im Westen

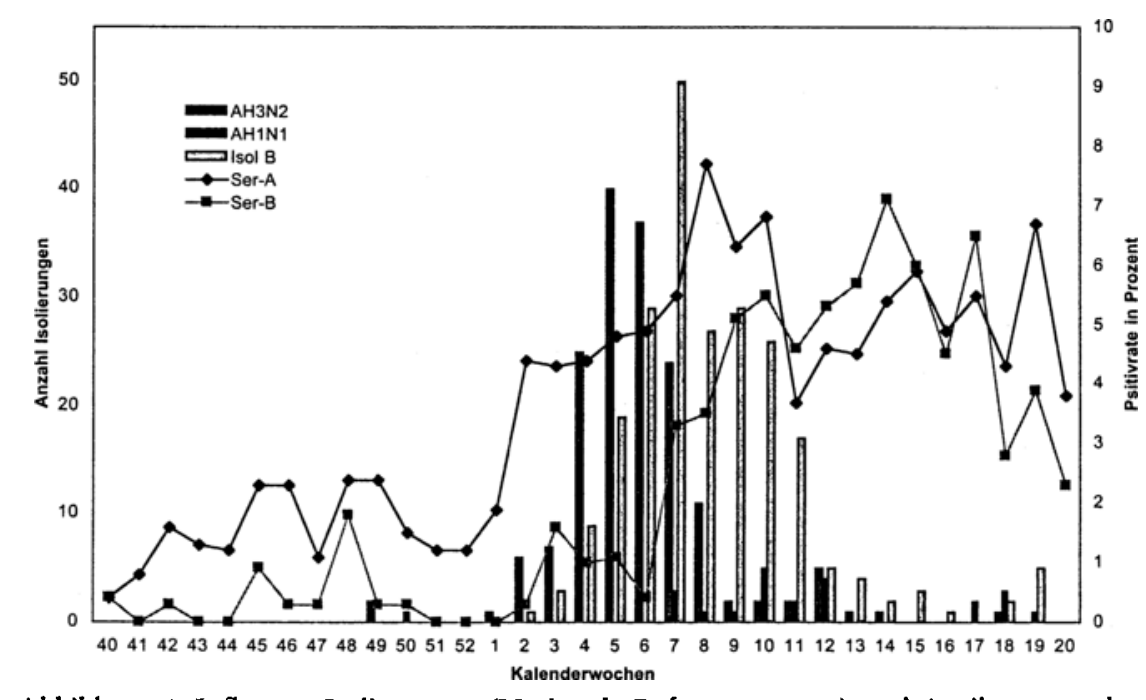

Abbildung 6: Influenza-Isolierungen (Nationale Referenzzentren) und Antikörpernachweise (Laborberichtssystem für respiratorische Erkrankungen RKI) 1996/97. 


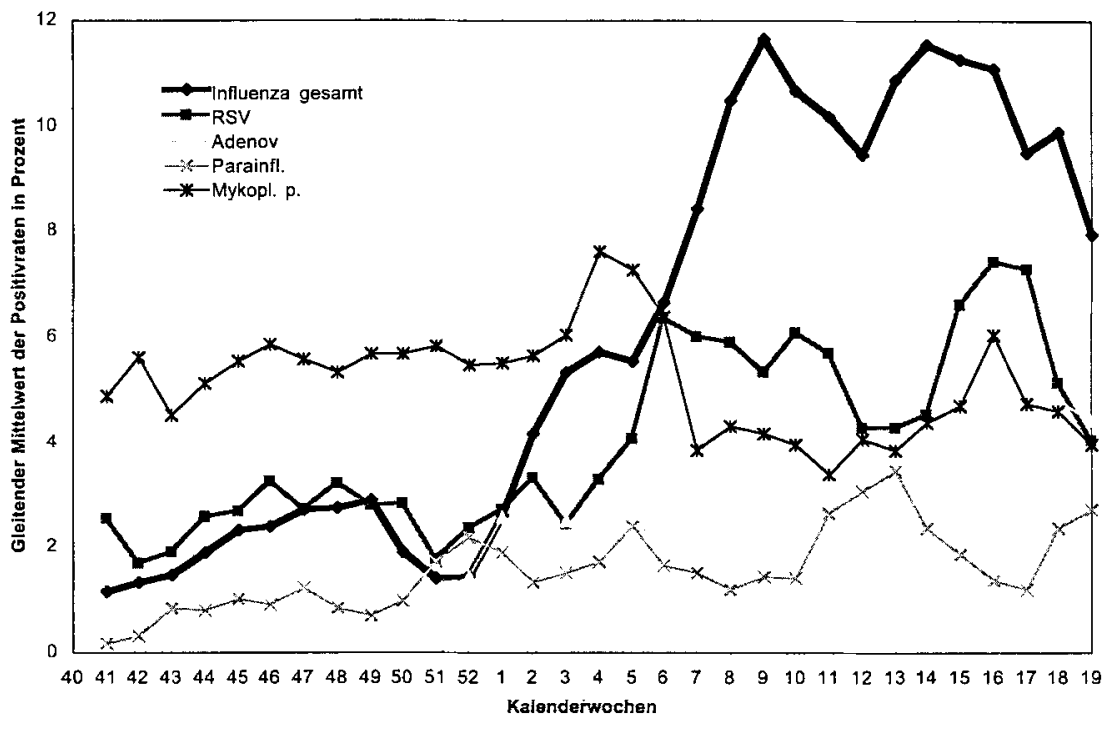

Abbildung 7: Gleitender Mittelwert (3) der Positivraten einzelner Erreger (Laborberichtssystem respiratorischer Erreger RKI).

nicht belegt werden. Zunehmende Isolierungszahlen bei einer steigenden Zah von Abstricheinsendungen ab der ersten KW 97 lassen eine leichte oder regionale Steigerung der Influenza-Aktivität über die Weihnachts- und Neujahrswoche nicht unplausibel erscheinen (Abb. 6).

Es folgte ein rascher Anstieg der Influenza-A(H3N2)-Nachweise mit einem Peak etwa in der fünften und sechsten $\mathrm{KW}$ sowie etwas zeitversetzt ein Anstieg der Influenza-B-Nachweise ab der zweiten KW mit einem Peak in der siebten bis neunten KW. Die Influenza$\mathrm{A}$ (H1N1)-Nachweise von der siebten bis zwölften $\mathrm{KW}$ deuten eine Phase leicht erhöhter Aktivität dieses Virussubtyps an. Leider war die Zahl eingesandter Proben in diesem Zeitraum bereits rückläufig, und die relativ wenigen Influenza-A(H1N1)-Isolierungen können nicht als Indikator für die Aktivität im Vergleich zu A(H3N2) und B gewertet werden. Es fällt auf, daß auch in diesem Jahr beide Influenzatypen und beide A Subtypen eine Phase erhöhter Aktivität erkennen ließen. Die bisherigen Feintypisierungen gaben keine Hinweise auf in dieser Beobachtungsphase zirkulierende neue Driftvarianten. Fast alle untersuchten Isolate entsprechen weitgehend den erwarteten und im Impfstoff enthaltenen Varianten. Bei der Influenza-A(H3N2) gab es einzelne Isolate, die eher der 1995/96 zirkulierenden Variante Johannesburg/33/94 entsprachen.
Laborberichtssystem für respiratorische Erkrankungen am RKI

Auch mit diesem Beobachtungssystem wurde $a b$ der zweiten $\mathrm{KW}$ ein moderater Anstieg der Influenza-A-Aktivität erfaßt. Nach einem Rückgang in der zehnten KW zeigt sich erneut ein Anstieg bis zur 15. KW. Ein erster Anstieg der Influenza-B-Aktivität wurde etwa ab der siebten $\mathrm{KW}$ bis zur neunten $\mathrm{KW}$ registriert. Nach einem Rückgang in der elften $\mathrm{KW}$ setzte sich die steigende Tendenz bis zur 14. KW fort. Die Positivraten für beide Typen weisen auf eine moderate Influenza-Aktivität hin. Die kumulative Positivrate für Influenza A und $B$ erreicht maximal etwa zwölf Prozent im Vergleich zu etwa 16 Prozent während der Influenza-A(H3N2)Epidemie zum Jahreswechsel 1995/96 (Ább. 7).

Im Vergleich zu den Vorjahren sind die geringen Positivraten für Parainfluenza und Adenoviren zu Beginn der Beobachtungszeit auffällig; die Hauptaktivität dieser Erreger ist dagegen in das Frühjahr 1997 verschoben. Eine Phase mit erhöhten RSV-Positivraten fällt mit dem Zeitraum der Influenza-Aktivität zusammen. Bemerkenswert ist auch ein zweiter Gipfel der RSV-Positivraten gegen Ende der Beobachtungszeit in der 15. KW. Die Positivraten der Mykoplasmennachweise waren im Herbst 1996 moderat, und es gab nur kurz nach dem
Jahreswechsel eine leichte Steigerung der Erkrankungsnachweise. Insgesamt zeigt sich bei keinem der mit diesem Beobachtungssystem registrierten Erreger eine auffällig hohe Positivrate.

\section{Diskussion}

Die für die Saison 1996/97 erwartete Influenza-A(H3N2)-Driftvariante Wuhan/359/95 hatte in einigen Ländern auf der Südhalbkugel (Neuseeland und Kolumbien) Epidemien mit im Vergleich zu den Vorjahren auffallend hohen Morbiditäten verursacht. Es wurde demnach auch in Europa damit gerechnet, daß sich möglicherweise eine epidemische Influenza-Aktivität aufbauen könnte. In der Bundesrepublik hat die mitrelschwere Influenza-A(H3N2)-Epidemie zum Jahreswechsel 1995/96 mit dem Subtyp Johannesburg/33/94 sehr ähnlichen Varianten möglicherweise für eine gewisse Immunität in der Bevölkerung gesorgt.

$\mathrm{Zu}$ Beginn der Beobachtungsperiode wurde in der Bundesrepublik eine auffallend niedrige ARE-Morbidität beobachtet, die etwa 20 Prozent unter den in anderen Jahren beobachteten Werten lagen. Dies steht möglicherweise auch in Zusammenhang mit den im Vergleich zu den Vorjahren auffallend geringen Positivraten für Parainfluenza- und Adenoviren, die von dem Laborberichtssystem für respiratorische Erkrankungen am RKI beobachtet wurden. Andererseits sind auch Auswirkungen der EBM Reform, die einer Selbstmedikation bei ARE förderlich sind, nicht auszuschließen. Da auch in der Schweiz und den Niederlanden die ARE- oder ILIMorbidität in diesem Zeitraum auffallend niedrig war, ist eine im Beobachtungssystem Deutschlands begründete Besonderheit eher unwahrscheinlich.

Zum Jahreswechsel hin (52. KW) überschritten die beobachteten Werte (ARE je 100 Praxiskontakte) erstmals die Basislinie. Dies wurde durch die mutmaßlich angestiegene Influenza-Aktivität besonders im Westen bewirkt. In diesem Zeitrahmen wurden aber auch (etwas verzögert) in dem Laborberichtssystem für respiratorische Erkrankungen des RKI steigende Positivraten bei Mykoplasmen, Adenoviren, RSV und Parainfluenza registriert. Dies spricht ebenfalls für die auch anhand der beobachteten Erkrankungen angezeigte Mischätiologie (AGI-Wochenbericht erste $\mathrm{KW}$ 1997) in diesem Zeitraum. Insgesamt 
zeigt jedoch keiner der erfaßten Erreger eine auffällig hohe Positivrate.

Bei diesen Überlegungen muß bedacht werden, daß der Anteil der einzelnen Erreger an den respiratorischen Erkrankungen, die zum Arztbesuch führen und damit von der AGI registriert werden, im einzelnen nicht bekannt ist $[3,4]$. Es bleibt auch offen, welchen Anteil die mit dem Berichtssystem des RKI nicht erfaßten Erreger, wie zum Beispiel die zu eher leichten Erkältungskrankheiten führenden Rhino- und Koronaviren [5], an den registrierten Erkrankungen haben. Dabei sind auch unterschiedliche Verhältnisse in den einzelnen Altersgruppen nicht unwahrscheinlich. Außer bei Kleinkindern dürfte jedoch durch die ausschließliche Erfassung von Arztkonsultationen eine deutliche Selektion zu gravierenderen Erkrankungen hin gegeben sein.

Besonders bei Kindern und Jugendlichen ging die Morbidität mit Beginn der Ferien zurück, was den Aufbau einer deutlich erhöhten Influenza-Aktivität zunächst stoppte. In der Aufbauphase einer Epidemie, wenn noch keine sehr hohen Morbiditäten erreicht wurden, kann dieses Phänomen bei Kindern und Jugendlichen häufiger beobachtet werden.

Bei der diesjährigen "Mischepidemie “ von Influenza A(H3N2)- und B-Viren, wobei der Gipfel der -A-Aktivität (vierte/fünfte $\mathrm{KW}$ ) etwas vor der Haupt-B-Aktivität (siebte/achte KW) lag, war der Morbiditätsverlauf in den Altersgruppen unterschiedlich.

Die in Relation zu den Fünf-bis 16jährigen hohe Morbidität unter den bis Vierjährigen läßr sich im Vergleich zum Vorjahr mit der deutlich erhöhten RSV-Aktivität erklären. Diese wurde in diesem Jahr parallel zu der Influenza-Aktivität beobachtet. Die Morbidität in den Altersgruppen der über 16jährigen ist in der Phase der Haupt-B-Aktivität am höchsten. Dies spricht ebenfalls für eine während der Epidemie 1995/96 aufgebaute Influenza-A(H3N2)-Immunität in diesen Altersgruppen. Zwar zeigen Immunseren gegen dic 1995/96 zirkulicrende Johannisburg-Variante nur geringe Titer (um drei bis vier Verdünnungsstufen vermindert) gegen das Hämagglutinin der diesjährigen $W_{u-}$ han-Variante, aber eine gewisse Kreuzreaktion ist noch vorhanden. Eine möglicherweise stärkere Kreuzreaktion weiterer Antikörper kann bei der natürlichen Immunität ebenfalls eine Rolle spielen.
In der neunten $\mathrm{KW}$ begann ein deutlicher Rückgang der Morbidität, der das Ende der Influenzawelle signalisiert. In diesen Zeitraum fallen aber einige Nachweise von Influenza $A(H 1 N 1)$, die cine regionale Aktivität überwiegend bei Kindern und Jugendlichen anzeigen. Besonders in diesen Altersgruppen war der Morbiditätsrückgang in der zehnten bis zwölften $\mathrm{KW}$ etwas verzögert. $\mathrm{Da}$ die Abstricheinsendungen in diesem Zeitraum rückläufig waren, spiegelt die Proportion der Isolierungen nicht unbedingt die tatsächliche Aktivität des (H1N1)-Subtyps wieder.

Zum Ende der Beobachtungsperiode ließen regionale Morbiditätsschwankungen eine weiterhin geringfügige lokale Influenza-Aktivität vermuten. Isolierungen aus diesem Zeitraum in Mecklenburg-Vorpommern und Sachsen sind weitere Indizien für eine bis in den Mai dauernde sporadische bzw. lokale Influenza-Aktivität. Von cinigen Arztpraxen wurden dabei auch Erkrankungen bei dem geimpften, bisher nicht erkrankten Klientel beschrieben. Die fünf bis sechs Monate nach der Impfung abgesunkenen Antikörpertiter scheinen nur noch einen geminderten Schutz zu bieten. $\mathrm{Da}$ es in den letzten Jahren Hinweise auf eine bis ins späte Frühjahr andauernde sporadische bis lokale Influenza-Aktivität gab, erscheint eine Impfung im November bis zum Dezember, oder besser bis zu den ersten Influenza-Ausbrüchen, durchaus sinnvoll.

In diesem Zusammenhang ist auch eine Ausdehnung der Beobachtungszeit bis zur 20. KW zu diskutieren. Eine Verbesserung der Kontinuität von $\mathrm{Ab}$ stricheinsendungen bis zum Ende der Beobachtungszeit ist ein wesentliches Ziel für die kommende Bcobachtungsphase.

\section{Arbeitsunfähigkeiten}

Die registrierten Arbeitsunfähigkeiten (AU) oder besser Erkrankungen, die häusliche Pflege erfordern, sind vielen weiteren Einflüssen unterworfen. Patientenverhalten (Arztbesuch auch bei leichteren Erkrankungen) und subjektive Einschätzung des Arztes und des Patienten spielen eine wesentliche Rolle und führen zu großen Unterschieden zwischen den Altersgruppen, bei denen keine AU-Bescheinigung notwendig ist und den Altersgruppen, die überwiegend in den Arbeitsprozeß eingebunden sind. Es ist im einzelnen auch nicht bekannt, ob die Registrierung der AU einheitlich und vollständig erfolgt, so daß eher von einer Untererfassung ausgegangen werden kann.

Der recht hohe und konstante Anteil der A-beitsunfähigkeiten an den registrierten ARE (etwa 40 Prozent) in den Altersgruppen der 16 - bis 60jährigen ist ein weiteres Indiz, daß zumindest in diesen Altersgruppen ein Arztbesuch erst bei etwas schwereren, behandlungsbedürftigen respiratorischen Infekten erfolgt. Somit kann vermutet werden, daß Rhinoviren und Koronaviren hier eine geringere Rolle spielen. Sie verursachen zwar häufiger respiratorische Infekte, diese verlaufen aber eher leicht und führen kaum zu Arztbesuchen.

Die Steigerung der Arbeitsunfähigkeiten auf etwa die Hälfte der mit Hilfe der Arztbesuche registrierten ARE während der Influenza-Aktivität erscheint gering, wenn man von einem hohen Anteil Influenza typischer Erkrankungen (Kopfschmerz, Gliederschmerz, Fieber über $38^{\circ} \mathrm{C}$ ) ausgeht. Auch während der A(H3N2)-Epidemie 1995/96 wurde dieses Verhältnis nicht überschritten. Andererseits bedeutet eine Steigerung des Anteils der AU um 10 Prozent bei einer Verdopplung der Erkrankungen eine Steigerung des Anteils um 20 Prozent bei den zusätzlichen Erkrankungen. Unter der Voraussetzung, daß das übrige Erkrankungsgeschehen etwa gleich bleibt und die Influenza zu zusätzlichen Erkrankungen führt, wären bei diesen zusätzlichen $\mathrm{Er}$ krankungen 60 Prozent Arbeitsunfähigkeitsfälle anzunehmen. Dies deutet unter Voraussetzung einer zuverlässigen Registrierung der Arbeitsunfähigkeiten auf einen recht hohen Anteil nicht influenzatypischer, aber dennoch vermutlich Influenza-bedingter Erkrankungen hin (da allein Fieber über $38^{\circ} \mathrm{C}$ zu einer $\mathrm{Ar}$ beitsunfähigkeit führen dürfte).

Die Zahl an "Arbeitsunfähigkeitsfällen « bei den anderen Altersgruppen läßt sich aufgrund einer möglicherweise nicht einheitlich gehandhabten Registrierung und Einstufung nur schlecht bewerten. Ein hoher Anteil leichterer Erkrankungen ist bei Kindern und speziell Kleinkindern anzunehmen. Bei den nicht in Einrichtungen lebenden über 60jährigen scheinen Arztbesuche während einer Erkrankungsphase, die häusliche Pflege indiziert, eher selten zu sein, und der Arztbesuch erfolgt (nach den Aussagen etlicher Ärzte und Ärztinnen) oft vor oder nach diesem Zeitraum. Ein Zusammenhang der Registrierung mit der Hausbesuchstätigkeit des Arztes ist 
nicht auszuschließen, wurde aber nicht untersucht.

\section{Hospitalisierungen}

Bei den unter Fünfjährigen fallen sowohl ein im Vergleich zu der Epidemie 1995/96 hoher Anteil Erkrankter als auch eine hohe Zahl an Hospitalisierungen auf. Der Anteil der Hospitalisierungen an den registrierten ARE ist während der Influenzawelle im Februar 1997 fast ein Drittel höher als während der insgesamt heftigeren Epidemie zum Jahreswechsel 1995/96. Die etwa in den Zeitraum der Influenza-Aktivität fallende starke RSV-Aktivität erscheint als schlüssigste Ursache. Dafür sprechen auch die Antigennachweise von RSV bei hospitalisierten Kindern am Klinikum der Christian-Albrechts-Universität Kiel und der Johann-WolfgangGoethe-Universität in Frankfurt am Main. Daneben ist aber auch ein etwas höherer Anteil an Influenza-assoziierten Hospitalisierungen durch die diesjährige A(H3N2)/Wuhan359/95-Driftvariante in dieser Altersgruppe nicht auszuschließen.

In der Altersgruppe der über 60jährigen kann trotz der nicht allzu stark ausgeprägten Morbiditätserhöhung im Februar/März 1997 ein deutlicher Anstieg der Hospitalisierungen beobachtet werden. Der Anteil an Hospitalisierungen an den registrierten ARE (normal etwa 0,7 - ein Prozent) steigt in diesem Zeitraum auf Werte bis etwa zwei Prozent. Während der Epidemie zum Jahreswechsel 1995/96 wurden Spitzenwerte von 2,5 Prozent beobachtet. $\mathrm{Da}$ auch in den Phasen erhöhter Influenza-Aktivität nicht alle Erkrankungen von Influenzaviren verursacht werden, dürfte das Risiko bei einer Influenzacrkrankung hospitalisiert zu werden, noch deutlich darüber liegen (drei Prozent und mehr bei den über 60jährigen). Der Einfluß der RSV-Aktivität auf die Hospitalisierungen bei über 60jährigen kann nicht abgeschätzt werden. Andere Studien [6, 7] weisen aber auf einen gewissen Einfluß von RSV auf Hospitalisierungen auch in dieser Altersgruppe hin.

\section{Todesfälle}

Der Anstieg der ARE-bedingten Todesfälle war in dieser Saison nicht sehr stark. Dennoch ist trotz des geringeren Anteils der über 60jährigen an der Morbidität (besonders im Vergleich zu März 1993 und Jahreswechsel 1995/96) eine signifikante (fünf Prozent-Niveau) Stcigerung der Todesfälle während der Influenza-Aktivität deutlich. In der To- desursachenstatistik dürfte dies jedoch kaum auffallen, $\mathrm{da}$ in diesem Zeitraum fast in jedem Jahr eine mehr oder weniger ausgeprägte Influenza-Aktivität auftritt.

In diesem Jahr sind zwar einige influenzabedingte Todesfälle auch bei Kindern und Jugendlichen bekannt geworden, was aber überwiegend der Aufmerksamkeit im Umfeld zu verdanken ist. Dabei konnten einige fulminante Verläufe und auch Todesfälle auf Influenza-B-Infektionen in Verbindung mit Sekundärinfektionen zurückgeführt werden. Der Influenza B werden allerdings generell cher leichtere Verläufe zugeschrieben.

Daneben sind, wie in jedem Jahr, weitere Todesfälle zu vermuten, bei denen keine Labordiagnostik durchgeführt wurde. Insgesamt ist die Influenza-Aktivität 1996/97 als moderat zu bezeichnen. Aufgrund der parallel zur InfluenzaAktivität aufgetretenen starken RSVAktivität ist eine auffällige InfluenzaMorbidität und -Mortalität für Kinder und Jugendliche nicht festzustellen.

\section{Allgemein}

Auch in der Beobachtungsphase 1996/97 haben die Daten der AGI eine frühe und zuverlässige Einschätzung der Lage ermöglicht. Bereits mit dem Auftreten erster relevanter InfluenzaAktivitäten in den Nachbarländern wurde eine zügige Fortführung der Impfkampagne mit dem Ziel befürwortet, sie in den nächsten Wochen abzuschließen (ab der 48. KW). Eine sporadische Aktivität wurde vor den ersten Isolierungen aufgezeigt. Bereits zwei Wochen vor dem Überschreiten der $\mathrm{Ba}$ sislinie $(50 . \mathrm{KW})$ wies die AGI auf eine hohe Wahrscheinlichkeit hin, daß die Influenza-Aktivität in den nächsten Wochen zunehmen könnte. Auch der Rückgang des ersten Morbiditätsaufbaues im Westen und die folgende Verzögerung der eigentlichen Influenzawelle bis in das nächste Jahr konnte sehr früh in der 51. KW erkannt werden. Zwei Wochen vor dem sprunghaften Morbiditätsanstieg bei Kindern und Jugendlichen war der Hinweis auf eine erneut steigende Influenza-Aktivität möglich. Diese frühzeitige und zuverlässige Einschätzung der Influenzasituation ermöglicht durchaus eine sichere Forrführung der Impfkampagne bis zum Beginn der Influenzawelle $[8,9]$. Natürlich kann auch darüber hinaus die Impfung fortgesetzt werden, jedoch mit steigendem Risiko, daß Personen - bei einer Impfung in der Inkubationszeit einer Influenza-Infektion - oder in dem Zeitraum von der Impfung bis zum Aufbau des Impfschutzes dennoch erkranken. Besonders wenn bei der Arztkonsultation zum Zwecke der Impfung ein ausgedehnter Kontakt mit bereits Erkrankten stattfindet, steigt die Gefahr einer gleichzeitigen Infektion.

Auch für den prophylaktischen oder therapeutischen Einsatz antiviraler Substanzen - wie das in Deurschland zugelassene und gegen Influenza A wirksame Amantadin, oder die in der Entwicklung befindlichen Neuraminidaseinhibitoren [10] sind zuverlässige Bewertungen der Influenza-Aktivität von großer Bedeutung.

Dennoch zeigen mehr als 15000 geschätzte Hospitalisierungen allein bei den über 60jährigen über das normale Maß hinaus (vierte bis neunte KW 1997), daß eine weitere Verbesserung der Durchimpfung der Risikogruppen notwendig ist $[11,12]$.

Die Verbreitung der einzelnen Influenzatypen und Subrypen war aufgrund der guten Zusammenarbeit mit den nationalen Referenzzentren, Landeshygieneinstituten und -untersuchungsämtern und einigen Labors gut erkennbar.

In den letzten beiden Jahren konnten einzelne Phasen mit erhöhter Aktivität für alle drei momentan relevanten Influenzatypen und -subtypen erkannt werden. Dies ist durchaus als ein Zeichen für die Qualizär der virologischen Surveillance zu sehen. Eine regionale Verlagerung der Aktivitätsschwerpunkte der einzelnen Typen und Subtypen läßt sich jedoch mit der derzeitigen Abstrichzahl nicht verfolgen.

Die Zahl der Isolierungen ist kein direktes Maß für die Verbreitung der Erkrankungen, insbesondere im Zusarnmenhang mit der unterschiedlichen Anzahl untersuchter Proben. Die Positivrate wäre ein geeigneterer Parameter, aber aus vielen Labors werden nur positive und selten und mit Verzögerung die in der jeweiligen Woche untersuchte Probenzahl mitgeteilt - es fehlt also dic Bezugsgröße. Aber auch bei der Positivrate müßte bei der vergleichsweise geringen Zahl von Abstrichen und der großen Zahl an störenden Einflüssen eine gewisse Unschärfe hingenommen werden. 
Auch das Laborberichtssystem für respiratorische Erkrankungen des RKI ermöglicht es nicht, die Verhältnisse der einzelnen Erreger an der Gesamtmorbidität ausreichend abzuschätzen. Auffällige Raten im Vergleich zu den Vorjahren und über mehrere Wochen anhaltende Steigerungen sowie auffällig hohe oder niedrige Positivraten geben aber bei sonst gleicher Handhabung in den Labors - wertvolle Hinweise auf das Krankheitsgeschehen.

\section{Literatur:}

[1] Lange, W., Uphoff, H., und Rasch, G.: Influenza 1995/96 - Ergebnisse zweier deutscher Überwachungssysteme. Bundesgesundhbl 10 (1996) 370-376.

[2] Uphoff, H.: Treffen des European Influenza Surveillance Scheme in Marburg. Bundesgesundhbl. 7 (1997) 237.

[3] Fleming, D. M., and Ayres, J. G.: Diagnosis and patrerns of incidence of influenza, influenza-like illness and the common cold in gen- eral practice. Jour. Royal Coll. Gen. Pract. 38 (1988) 159-162.

[4] Dab, W., Cohen, J. M., and Hannoun, C.: A new Influenza surveillance system in France: The ile-de-France »Grog “. 2. Validicy of Indicators. Eur. J. Epidemiol. 7, 6 (1991) 579-587.

[5] Tyrell, D. A. J.: Erkältungskrankheit. Gustav Fischer Verlag 1996.

[6] Dowell, S. F., Anderson, L. J., Gary, H. E, et al.: Respiratory Syncytial Virus Is an Impor tant Cause of Community-Acquired Lower Respiratory Infection among Hospitalized Adults. The Journal of Infectious Diseases 174 (1996) 456-462.

[7] Fleming, D. M., and Cross, K.W.: Respiratory syncytial virus or Influenza? The Lance 342 (1993) 1507-1510:

[8] Fleming, D. M.: The prediction of epidemics of respiratory Infections. 7th European Meet ing of Influenza and its Prevention. Eur. J Epidemiol. 10 (1994) 481-484.

[9] Zuckemann, M. A., Cox, R., Taylor, J. Wood, J., Haaheim, L., and Oxford, J.: Rapid immune response to Influenza vaccination Lancet 342 (1993) 1113.
[10] Itzenstein von, M.: Design and synthesis of influenza virus sialidase inhibitors: Novel antiinfluenza drugs. Options for the control of Influenza III 1996; Elsevier Science B.V.

[11] Carrat, F, and Valleron, A. J.: Influenza mor tality among the eiderly in France, 1980-90: how many death may have been avoided through vaccination?. Jour. of Epid. and Community Health 49 (1995) 419-425.

[12] Nichol, K. L., Margolis, K. L., Wuorenma, J., and Sternberg, T.: The efficacy and cost effectiveness of vaccination against Influenza among elderly persons living in the community. The New England Jour. of Medicine 22 (1994) 778-784.

Anschrift der Verfasser

Dr. Helmut Uphoff, Arbeitsgemeinschaft Influenza, Schumarke 4, 35037 Marburg/Lahn, Dr. Rolf Heckler, Landesgesundheitsamt Hannover, Rösebeckstr. 4-6, 30449 Hannover, Dr. Gernot Rasch, Dr. Brunhilde Schweiger, Dr. Werner Lange, Robert Koch-Institut, Nordufer 20, 13353 Berlin.

\section{Toxizität und Exposition einiger Bestandteile von Lebensmittelverpackungen*}

An einigen praktischen Beispielen von Stoffen, die zur Herstellung von Lebensmittelverpackungen dienen und die in jüngster Zeit wegen möglicher östrogener Wirkungen in der Offentlichkeit diskutiert worden sind, soll gezcigt werden, welche Daten zur Toxizität und Exposition vorliegen und welche $\mathrm{Be}$ wertungen aus diesen Daten abgeleitet wurden.

\section{2,2-Bis (4-hydroxyphenyl) propan (Bisphenol A)}

Bisphenol A (BPA) wird als Ausgangs material zur Herstellung von Innenlacken für Konservendosen und des Kunststoffs Polycarbonat verwendet. Aus diesem Kunststoff werden u. a. auch Trinkflaschen für Babys hergestellt. Die Migration von BPA aus diesen Materialien ist sehr gering und die Exposition beträgt auch unter "worst case«-Annahmen nur ca. $1 \mu \mathrm{g} / \mathrm{kg}$ Körpergewicht.

\footnotetext{
Vortrag gehalten auf der Fortbildungsveranstaltung für den Öffentlichen Gesundheits dienst vom 5. bis 7. 3. 1997 in Berjin
}

Der Wissenschaftliche LebensmittelAusschuß der EU, der die wissenschaftlichen Grundlagen für die Richtlinien der Gemeinschaft auf dem Gebiet der Materialien im Kontakt mit Lebensmitteln schaffe, hat auf der Basis der vorhandenen toxikologischen Daten einen TDI-Wert (tolerable daily intake) von $0,05 \mathrm{mg} / \mathrm{kg}$ Körpergewicht festgelegt.

Von BPA ist schon seit langem bekannt $\mathrm{da} ß$ es eine östrogene Wirkung im Tierversuch besitzt; allerdings ist es dazu erforderlich, bis in den toxischen Bereich hinein zu dosieren. Für BPA gibt es auch sehr umfangreiche Tierversuche auf dem Gebiet der Teratogenität und Reproduktionstoxizität, und für diese Endpunkte wurden $50 \mathrm{mg} / \mathrm{kg}$ Körpergewicht als niedrigste Dosis ohne Wirkung ermittelt.

Diese Gegenüberstcllung zeigt, daß zwischen der Exposition des Verbrauchers und den Wirkschwellen im Tierversuch ein sehr großer Abstand besteht und es derzeit keine Hinweise auf eine mögliche Gesundheitsgefährdung des Verbrauchers gibt.

\section{Phthalsäureester}

Vor einiger Zeit gab es Meldungen aus England über das Vorkommen kleiner Mengen von Phthalsäureestern in Milchpulver zur Herstellung von Säug lingsnahrung. Darunter waren auch $\mathrm{Di}^{-}$ n-butylphthalat (DBP) und Butylbenzylphthalat (BBP), die in einigen Invitro-Untersuchungen eine schwache östrogene Wirkung gezeigt haben. Die gefundenen Phthalat-Gehalte entsprachen einer Aufnahmemenge für den Säugling von $0,014 \mathrm{mg} / \mathrm{kg}$ Körpergewicht für DBP und $0,09 \mathrm{mg} / \mathrm{kg}$ Körpergewicht für BBP.

Dies hat dazu geführt, daß auch in Deutschland sowohl durch die Überwachungsbehörden als auch durch die Industrie Untersuchungen an verschiedenen Arten von Säuglingsnahrung durchgeführt wurden. Sie brachten das insgesame erfreuliche Ergebnis, daß die Mengen an Phthalsäureestern in Säuglingsnahrung sehr gering sind. Es hat sich aber auch gezeigt, daß diese Stoffe nicht aus der Verpackung stammen, denn die Kunststoff-Einlage in dem Schraubverschluß enthielt keine Phtha- 